\title{
Microscopy101
}

\section{A Fast, Simple, and Safe Way to Prepare Paraformaldehyde Solutions}

\section{E. Ann Ellis}

Microscopy and Imaging Center, Texas A\&M University, 2257 TAMU, College Station, TX 77843

eann.ellis@worldnet.att.net

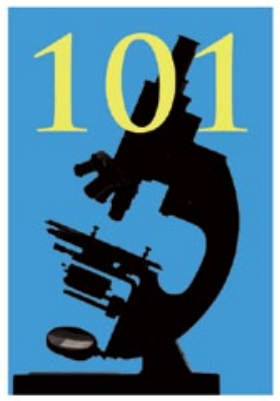

Depolymerized paraformaldehyde solutions have been used in fixation of specimens for electron microscopy since Karnovsky [1] proposed the combination of glutaraldehyde and paraformaldehyde for improved specimen preservation. Preparation of depolymerized paraformaldehyde solutions requires the use of heat (approximately $60^{\circ} \mathrm{C}$ ) and raising the $\mathrm{pH}$. Most labs place a heating stir plate in the hood and then heat to $60^{\circ} \mathrm{C}$ followed by adding drops of concentrated sodium hydroxide solution until the solution of paraformaldehyde clears. A precise temperature of $60^{\circ} \mathrm{C}$ is not required; raining the $\mathrm{pH}$ by adding sodium hydroxide is the most important action in achieving depolymerization and clearing of the paraformaldehyde solution.

The following time-saving and safe protocol has been used in my lab for a number of years:

1. Weigh out the required amount of paraformaldehyde, and put it into an appropriate size flask to hold the final solution of depolymerized paraformaldehyde. Add 1-2 pellets of sodium hydroxide. Place this flask in the hood.

2. Heat the required volume of deionized water for 1 minute on high in the microwave.

3. In the hood, add the heated water to the flask of paraformaldehyde and sodium hydroxide pellets. Swirl the flask until the solution clears, which usually takes 1-2 minutes.

4. The solution of depolymerized paraformaldehyde is now ready for use in preparing the fixative.

The total time for this preparation is no more than 5 minutes, a time savings of about 30 minutes. In addition, there is no need for a hot plate. VT

\section{References}

[1] MJ Karnovsky, J Cell Biol 27 (1965) 137A.
PELCO

BioWave ${ }^{\oplus}$ Pro

Fast, Low Temperature Microwave Tissue Processing

- Rapid Specimen Turnaround

- Low Temperature Environment

- Programmable Processing

- Consistent Quality Results

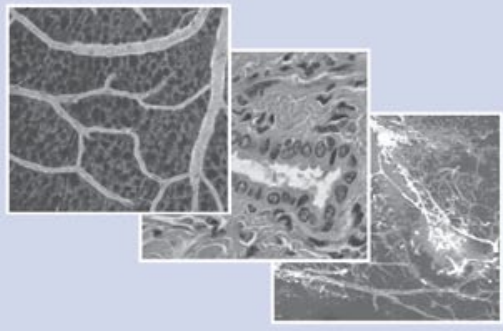

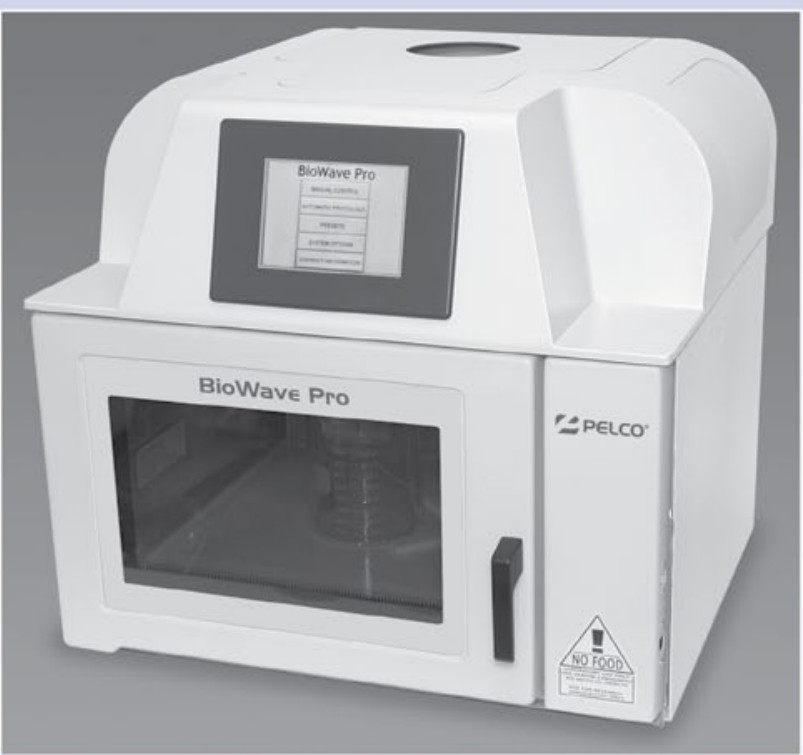

Application kits for paraffin processing, decalcification, confocal, light and electron microscopy are available.

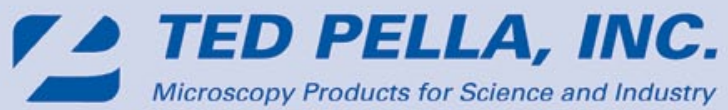

sales@tedpella.com・Tel: 800-237-3526•www.tedpella.com 
DIATOME

\section{diamond knives}

Development, Manufacturing, and Customer Service since 1970

What have we achieved in this period?

ultra $45^{\circ}$ the first diamond knife with an absolutely score-free, hydrophilic cutting edge.

semi the first diamond knife for alternating sectioning ultrathin/semithin.

cryo the diamond knife for sectioning at low temperature.

histo the first diamond knife for semithin sections for light microscopy.

ultra $35^{\circ}$ the diamond knife for optimized sectioning results in almost all applications.

STATIC LINE || the ionizer for eliminating electrostatic charging in ultramicrotomy.

cryo-P a cryo knife with a patented platform for section pick up.

cryo immuno the optimized cryo diamond knife for the Tokuyasu technique.

ultra sonic the oscillating diamond knife for room temperature sectioning.

cryotrim 45 and 25 optimizing trimming with diamond blades.

ultra AFM \& cryo AFM the first diamond knives for AFM at room and low temperatures.

cryo $25^{\circ}$ for sectioning frozen hydrated specimens.

What services can we offer you?

I Technical assistance in all fields of ultramicrotomy.

I Free sectioning tests for all types of samples.

I Make use of our many years of experience in perfecting our knives.

I Custom knives, tools, and boats.

I Special purchase programs.

Workshops and training.

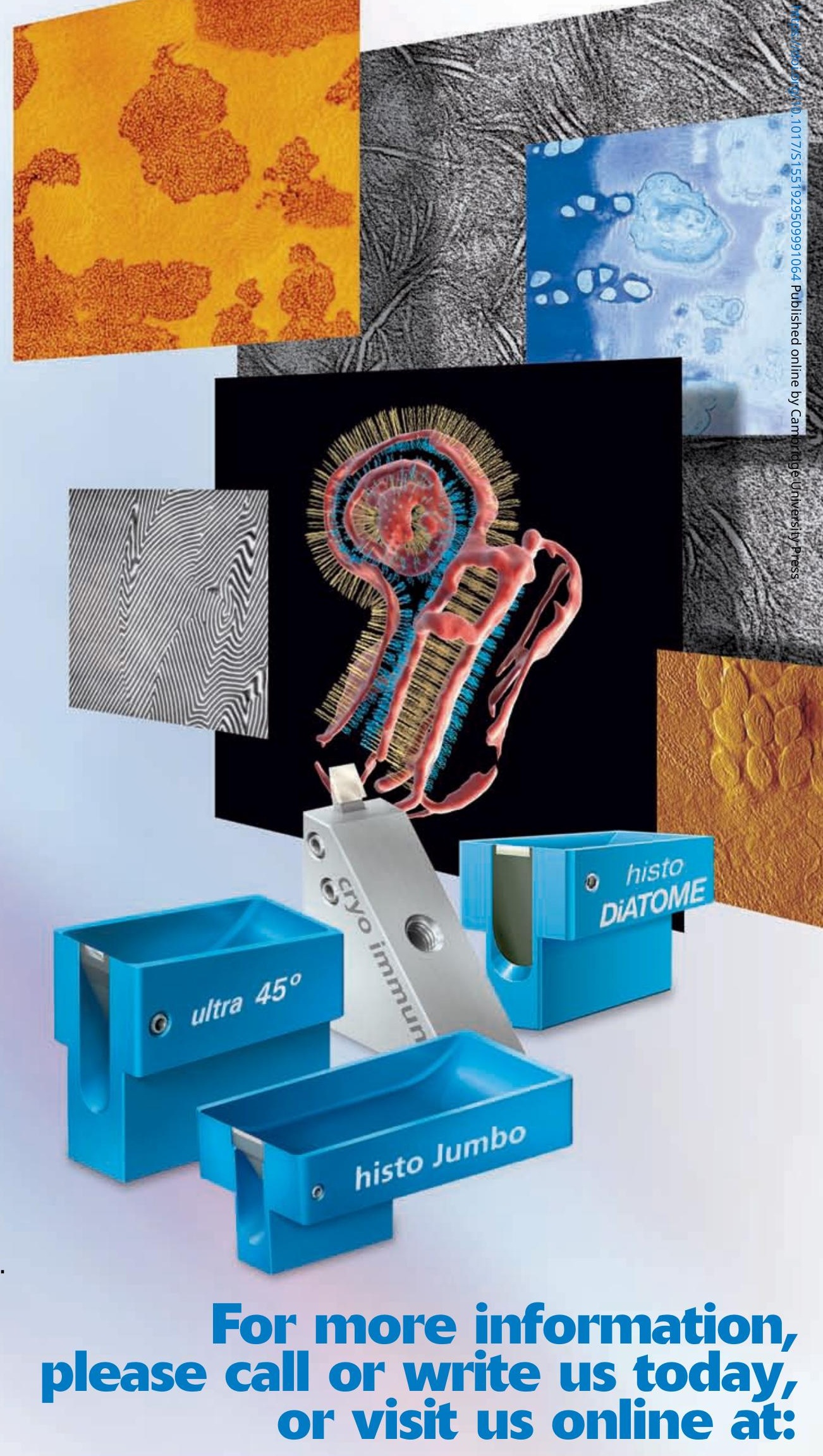

\section{wwwemsdiasum.com}

\section{DIATOME}

for all your sectioning requirements

\section{P.O. Box $410 \bullet 1560$ Industry Rd.} Hatfield, Pa 19440

(215) 412-8390 • Toll Free: 1-(800) 523-5874

Fax: (215) $412-8450$ or 8452

email: sgkcck@aol.com•stacie@ems-secure.com 